\title{
Assessing the Adaption of Stochastic Clearing Procedure to a Hydro-penetrated Market
}

\author{
Nilufar Neyestani, Filipe J. Soares \\ Centre for Power and Energy Systems \\ INESC TEC \\ Porto, Portugal \\ nilufar.neyestani@inesctec.pt
}

\author{
Rui Alves, Francisco S. Reis, Ricardo Pastor \\ R\&D NESTER \\ Lisbon, Portugal
}

\begin{abstract}
Vast increase of renewable energy resources' (RER) share in total electricity production have led to evolving studies regarding different aspects of renewables integration. Other than their effects on network, the electricity markets are also affected by uncertain behavior of RERs in the market place. Hence, new approaches for market clearing are investigated. One of the possible solutions is the deployment of stochastic market clearing. However, the adaption of new market models should consider different market characteristics. As a result, this paper assesses the adaption of stochastic market in a hydropenetrated system. The co-optimized energy and reserve schedule in the day-ahead time frame is derived using the mixed integer linear programming (MILP). The model is tested with Portuguese electricity market data as a real-case of hydropenetrated system.
\end{abstract}

Index Terms-Hydro units, market clearing model, probabilistic consumption, stochastic programming, wind power.

\section{NOMENCLATURE}

Subscripts
$d, d^{\prime}$
$i, k$
$s$
$\omega$
$w$
$j, j^{\prime}$
$t$

Loads running from 1 to $N D$

Generator units running from 1 to $N G$

Wind scenarios running from 1 to $N S$

Load scenarios running from 1 to $N \omega$

Wind generator units running from 1 to $N W$

Grid nodes running from 1 to $N N$

Time interval running from 1 to $N T$

Superscripts

$\begin{array}{ll}\text { Agg } & \text { EV aggregator } \\ \text { curtail } & \text { Wind curtailment option } \\ \text { DW } & \text { Downward reserve } \\ \min / \max & \text { Minimum and Maximum Value } \\ \text { UW } & \text { Upward reserve }\end{array}$

Parameters and Variables

$B \quad$ Susceptance

D Total stochastic consumption in each scenario

ND Total number of loads

$N G \quad$ Total number of generators

NN Total number of grid nodes

NT Total number of hours

This work is financed by the ERDF - European Regional Development Fund through the Operational Programme for Competitiveness and Internationalisation - COMPETE 2020 Programme, and by National Funds through the Portuguese funding agency, FCT - Fundação para a Ciência e a Tecnologia, within projects SAICTPAC/0004/2015- POCI-01-0145-FEDER-016434 and SusCity - MITPTB/CS/0026/2013."

\author{
NW Total number of wind units \\ $N \omega$ \\ $p, P$ \\ $r, R$ \\ $S \quad$ Binary variable determining the location of \\ each unit \\ $u \quad$ Binary variable indicating the acceptance of \\ units in the day-ahead market \\ $W \quad$ Total wind unit production in each scenario \\ $\delta \quad$ Node angle \\ $\rho \quad$ Probability \\ $\pi \quad$ Bidding price
}

Remark I Capital letters denote parameters and small ones denote variables.

\section{INTRODUCTION}

The electrical network operation has been the subject of many changes since the integration of renewable resources specially wind and solar units. On the other hand, with the growing penetration of electric vehicles the level of uncertainty is increasing more than before. Various studies tried to solve the concerns regarding the integration of intermittent resources in the system. With the provided solutions, the penetration of such resources has increased; hence, the system operators relied more on renewables. Moreover, governmental and social motivations and promotions have also encouraged the massive deployment of renewables. Different incentive schemes have been employed to promote the investors to build renewable power plants. The feed-in tariff (FIT) approach has been one of the most popular solutions to increase the share of renewable penetration and guarantee their payback.

The electricity markets, like any other market, encounter various uncertainties in their operation. The uncertainty of price has been one of the most affecting issues on the liberalized markets. However, when dealing with the conventional generators and loads, the level of uncertainty imposed from generation and demand side is not as much as the case where the renewable resources are introduced. From the electricity market point of view, in order to make 
renewable resources more economically viable, the regulations regarding the renewable resources need to be revised. The main step is to make the renewable generator owners accountable for the uncertainty they bring into the power system. The stochastic behavior of the renewables in the power system, impose an extra cost to the system operation especially in real-time. Therefore, it is necessary to change the conventional approach towards the stochastic resources participation in the market. The solution is to move from the deterministic market clearing approach to the stochastic market operation where the stochasticity of the renewables would also be taken into account in the market procedure.

There are several studies that have investigated the intermittency of wind generators in the energy market. However, addressing the compensation of uncertainty in the market clearing stage has been the interest of a few previous studies. The Stochastic day-ahead clearing formulations have been proposed by [1] and [2]. In [3] a model is proposed to clear an energy-only electricity market with intermittent participants. It is assumed that both suppliers and consumers are bidding in the day-ahead and real-time. The study in [4] formulates a market-clearing model for the co-optimization of energy and reserve through a two-stage problem. Other studies such as [5] and [6] propose a bilevel approach to solve the stochastic market. They used an equilibrium model to optimize the reserve mobilization in response to stochastic resources intermittency.

Considering the probabilistic participation of market agents in the clearing procedure, the risk that is imposed to the market/system operator can have significant effects. As a result, several existing studies have considered risk as an affecting factor in modelling the stochastic market clearing procedure. In [7], the strategy for the wind generators to offer in the market is proposed. Some of other existing studies have considered the conditional value at risk $(\mathrm{CVaR})$ as the system risk measure [8] - [10]. For example, the study in [8] has modelled the cost occurred due to the deviation of wind production using $\mathrm{CVaR}$ where it defined the maximum acceptable level of risk for the system based on the probabilities of wind scenarios. In [9] the CVaR measure is employed to consider the risk constraint in the stochastic unit commitment problem while the authors in [10] use the same approach for a stochastic optimal power flow with probabilistic wind offers.

As the operating conditions of the generating units may bring non-convexities to the model, the authors in [11] addressed such conditions such as start-up costs and the on/off status of generators and their minimum power outputs in their proposed model.

A new method for the European balancing markets is proposed in [12] where a "flexible reserve" is considered to address the unpredictability of wind generation. The model is proposed for a day-ahead balancing market and is applied to price taker generating units. Moreover, the authors in [12] tested their model with a simplified Spanish market model having in mind the level of wind penetration in Spain. The implementation of stochastic market model for the reserve market was tested in Swiss electricity market as described in [13]. A probabilistic approach for the offers submitted to the reserve market is proposed in [13] stating that the model has been used in Swiss since 2014 and have shown significant improvement in market results.

Although the previous studies addressed several aspects of the stochastic market clearing model regarding the mathematical model, market settlement, and cost recovery solutions, they are more focused on the reserve activation from generation side. It is mainly considered that the generators are offering the reserve participation while in practice, the downward reserve participants are mainly on the demand side. The case is more dominant when the pumping units are available in the system. The authors in [14] have considered the reserve mobilization for compensation of wind deviations, however, the main assumption in their work is that the demand side's flexibility is an option which should be evaluated in sense of being profitable for those who volunteer in the reserve activation.

The study presented in this paper, takes into account the fact that in a hydro-penetrated system, the pumping units willingly participate in downward reserve regardless of the possible profit achievement, but mainly because of their physical constraints. Therefore, in this paper a stochastic model is proposed for a two-stage stochastic market model where both generation and demand side participates in the reserve market. It is assumed that the amount of required reserve for compensating the deviations in wind production is provided both from the generators and loads that offer in the reserve market. The model is proposed for the day-ahead schedule of co-optimizing energy and reserve market. The results discuss the efficiency of the model comparing to the previously proposed models as well as testing it with real data of Portuguese electricity market.

The rest of the paper is organized as follows. In Section II the mathematical model of the market clearing procedure is explained. Section III provides the data of Portuguese market as well as the case-studies and results. Finally, Section IV concludes the paper.

\section{Market Clearing Mathematical Model}

The proposed model is a day-ahead market clearing problem that takes into account the expected cost of compensating intermittent participants' deviations with the activation of the reserve. The objective of the market operator is to minimize the total cost which includes the day-ahead cost and expected balancing cost. The mathematical model is presented in (1). The first bracket denotes the day-ahead cost while the second one represents the expected cost of reserve activation due to the probabilistic behavior of PEV aggregator or wind units.

$$
\begin{aligned}
\operatorname{Min} & \sum_{t=1}^{N T}\left[\sum_{i=1}^{N G} \pi_{i, t} p_{i, t}-\sum_{d=1}^{N D} \pi_{d, t} p_{d, t}\right]+ \\
& \sum_{\omega=1}^{N \omega} \rho_{\omega}\left[\sum_{t=1}^{N T}\left(\sum_{i=1}^{N G}\left(\pi_{i, t}^{U W} r_{i, \omega, t}^{U W}-\pi_{i, t}^{D W} r_{i, \omega, t}^{D W}\right)+\sum_{d=1}^{N D}\left(\pi_{\mathrm{d}, t}^{U W} r_{d, \omega, t}^{U W}-\pi_{\mathrm{d}, t}^{D W} r_{\mathrm{d}, \omega, t}^{D W}\right)\right)\right]
\end{aligned}
$$


The above mentioned model is subject to balancing equation constraints, both on the day-ahead stage and the balancing stage of the market. In (2), it is shown that the total production from conventional units as well as wind should provide the probabilistic load of EV aggregator $\left(p_{e}\right)$ and other loads. A DC power flow model is employed in this study.

a) Power balance constraint at the day-ahead stage: For the balancing stage, the total upward/downward activated reserve should compensate the deviations occurred in wind production or EV aggregator consumption. Note that $S$ is a binary variable determining whether a unit is located on node $j$ or not.

$\sum_{i} S_{i} p_{i, t}+\sum_{w} S_{w} p_{w, t}-\sum_{d} S_{d} p_{d, t}-\sum_{j} B_{j j^{\prime}}\left(\delta_{j, t}-\delta_{j^{\prime}, \mathrm{t}}\right)=0, \quad \forall \mathrm{t}$

b) Power balance constraint at the balancing stage: This equation is the main representation of the model's assumptions. As mentioned before, the deviations caused by uncertain generation or load should be compensated by the amount of activated reserve (upward or downward). This means that the amount of wind that has been dispatched in the day-ahead is compared with the available amount of wind in each scenario. If there would be any deviation between these two amounts, some solutions are foreseen to compensate this deviation: mobilization of reserve up/down from generators, mobilization of reserve from the load, and wind curtailment option. It should be noted that by adding the curtailment option to the model, the market operator would have the opportunity to spill the excess amount of wind instead of mobilizing the reserve.

$$
\begin{aligned}
& \sum_{i} S_{i}\left(r_{i, \omega, t}^{U W}-r_{i, \omega, t}^{D W}\right)+\sum_{w} S_{w}\left(W_{w, \omega, t}-p_{w, t}-p_{w, \omega}^{c u r t a i l}\right)+\sum_{d} S_{d}\left(r_{\mathrm{d}, \omega, t}^{U W}-r_{\mathrm{d}, \omega, t}^{D W}\right) \\
& +\sum_{j^{\prime}} B_{j j^{\prime}}\left(\delta_{j, t}-\delta_{j, \omega, t}-\delta_{j, t}+\delta_{j^{\prime}, \omega, t}\right)=0 \quad \forall \omega, \forall \mathrm{j}, \forall t
\end{aligned}
$$

c) Minimum/Maximum generation/load constraint: The minimum and maximum limit of generator and demand units for all conventional and uncertain units should be taken into account as in (4) and (5). The binary variable $u$ is used in the model to enforce the limitation on the minimum and maximum of the unit whenever it is accepted in the market.

$$
\begin{gathered}
u_{i, t} P_{i}^{\min } \leq p_{i, t} \leq u_{i, t} P_{i}^{\max }, \quad \forall i, \forall t \\
0 \leq p_{w, t} \leq P_{w}^{\max }, \quad \forall w, \forall t \\
u_{d, t} P_{d}^{\min } \leq p_{\mathrm{d}, t} \leq u_{d, t} P_{d}^{\max }, \quad \forall d, \forall t
\end{gathered}
$$

d) Upward/Downward reserve offer constraint: The maximum available offer for participating in the upward/downward reserve is determined by the generator or load through their offers in the balancing market. It should be noted that these constraints should be met in all hours, in all scenarios and for all generating units and loads.

$$
\begin{aligned}
& 0 \leq r_{i, \omega, t}^{U W} \leq R_{i, t}^{U W, \text { max }}, \quad \forall i, \forall \omega, \forall t \\
& 0 \leq r_{i, \omega, t}^{D W} \leq R_{i, t}^{D W, \text { max }}, \quad \forall i, \forall \omega, \forall t \\
& 0 \leq r_{\mathrm{d}, \omega, t}^{U W} \leq R_{\mathrm{d}, t}^{U W, \max }, \quad \forall d, \forall \omega, \forall t \\
& 0 \leq r_{\mathrm{d}, \omega, t}^{D W} \leq R_{\mathrm{d}, t}^{D W, \max }, \quad \forall d, \forall \omega, \forall t
\end{aligned}
$$

e) Upward reserve activation constraint: When activating the upward reserve for the generating unit, it should be noted that the sum of each unit's dispatch in the day-ahead and the amount of activated reserve in the balancing stage should not exceed the limit of the maximum possible generating of each unit (11).

$$
p_{i, t}+r_{i, \omega, t}^{U W} \leq u_{i, t} P_{i, t}^{\max }, \quad \forall i, \forall \omega, \forall t
$$

Unlike generating units, for the activation of upward reserve for loads, it should be considered that the subtraction of dispatched load in the day ahead and the amount of upward reserve activation should be greater than zero (12). This difference between constraints of upward reserve of generating units and loads occurs due to different functionality of upward reserve in generators and loads.

$$
p_{d, t}-r_{d, \omega, t}^{U W} \geq u_{d, t} P_{d}^{\mathrm{min}}, \quad \forall d, \forall \omega, \forall t
$$

f) Downward reserve activation constraint: For activation of downward reserve, it should be noted that the total downward activation of each unit should not surpass the amount that has been dispatched in the day-ahead stage (13). Moreover, for the downward reserve activation of the loads, the maximum limit of each load unit should be considered (14).

$$
\begin{gathered}
p_{i, t}-r_{i, \omega, t}^{D W} \geq u_{i, t} P_{i}^{\min }, \quad \forall i, \forall \omega, \forall t \\
p_{i, t}+r_{i, \omega, t}^{D W} \leq u_{d, t} P_{d, t}^{\max }, \quad \forall d, \forall \omega, \forall t
\end{gathered}
$$

g) Maximum curtailment constraint: It is considered that, if necessary, up to the maximum amount of produced wind in a scenario can be curtailed to avoid the excess cost of reserve activation.

$$
0 \leq p_{w, t}^{\text {curtail }} \leq W_{w, t}
$$

h) Power flow constraints: The power flow of the lines with the DC power flow procedure should meet the line limits both in the day-ahead stage and in the balancing stage. Note that node angles will be different in each scenario. 


$$
\begin{gathered}
-f_{j j^{\prime}}^{\max } \leq B_{j j^{\prime}}\left(\delta_{j, t}-\delta_{j^{\prime}, t}\right) \leq f_{j j^{\prime}}^{\max }, \quad \forall j, \forall t \\
-f_{j j^{\prime}}^{\max } \leq B_{j j^{\prime}}\left(\delta_{j, \omega, t}-\delta_{j^{\prime}, \omega, t}\right) \leq f_{j j^{\prime}}^{\max }, \quad \forall j, \forall t, \forall \omega
\end{gathered}
$$

i) Constraint to avoid simultaneous upward/downward reserve: Considering the practical aspects of system operation, it is known that the market operator prevents the activation of upward reserve for some units while other units are providing downward reserve. This means that the simultaneous activation of upward and downward reserve for units should be avoided and it should be maintained for both generation and load. This constraint is formulated in the model as shown in (18) and (19) for generators and loads, respectively.

$$
\begin{gathered}
r_{i, \omega, \mathrm{t}}^{U W} \cdot r_{\mathrm{k}, \omega, \mathrm{t}}^{U W}=0, \quad \forall t, \forall \omega, \forall k \in\{N G-i\} \\
r_{\mathrm{d}, \omega, \mathrm{t}}^{U W} \cdot r_{d^{\prime}, \omega, \mathrm{t}}^{D W}=0, \quad \forall t, \forall \omega, \forall d^{\prime} \in\{N D-d\}
\end{gathered}
$$

As it is observed, the equations (18) and (19) are nonlinear hence the problem would be a nonlinear one. In order to solve the problem, a mixed integer linear programming (MILP) is used and the nonlinear equations are converted to linear ones using the equivalent mixed-integer linear formulation and thus, branch-and-cut software such as CPLEX can be employed to solve the model. For example, consider a large enough constant $M$, Eq. (18) can be equivalently formulated in a linear format as follows

$$
\begin{gathered}
r_{i, \omega, \mathrm{t}}^{U W} \leq M \alpha_{\omega, \mathrm{t}} \\
r_{\mathrm{k}, \omega, \mathrm{t}}^{U W} \leq M\left(1-\alpha_{\omega, t}\right)
\end{gathered}
$$

where $\alpha$ is a binary variable.

\section{NUMERICAL RESULTS AND ANALYSIS}

The model presented in the previous section has been tested with the real data from the Portuguese electricity market. Some of the data that are used in this simulation are publicly available on [15] and [16]. The existing day-ahead market bids as well as the regulating reserve bids are derived from the aforementioned references, however, for making the market agents in both energy and reserve market bids correspondent, some justifications has been made. It should also be noted that for the sake of simplicity no network has been considered in the case-studies. The complex biddings of generating units have also been neglected because of no available data on the matter.

The main characteristic of the Portugal's electricity market is that it has high levels of hydro generation participating in the market. Therefore, the level of flexibility both from supply and demand side is rather high. As a result, several case studies have been performed to analyze the consequences of deploying the stochastic market clearing model in a hydropenetrated network.
TABLE I. WIND AGENT BIDS IN CASES I AND II

\begin{tabular}{|l|c|c|c|c|c|c|c|}
\cline { 2 - 7 } \multicolumn{1}{c|}{} & \multicolumn{2}{c|}{ Scenario \#1 } & \multicolumn{2}{c|}{ Scenario \#2 } & \multicolumn{2}{c|}{ Scenario \#3 } & \multirow{2}{*}{$\begin{array}{c}\text { Expected } \\
\text { Value }\end{array}$} \\
\cline { 2 - 7 } \multicolumn{1}{c|}{} & $\boldsymbol{M W}$ & Prob. $^{*}$ & $\boldsymbol{M W}$ & Prob. & MW & Prob. & 619 \\
\hline $\begin{array}{l}\text { Type } \\
\text { I/Diverged }\end{array}$ & 205 & 0.2 & 650 & 0.6 & 940 & 0.2 & 600 \\
\hline $\begin{array}{l}\text { Type } \\
\text { II/Converged }\end{array}$ & 460 & 0.3 & 622 & 0.4 & 710 & 0.3 & 600 \\
\hline
\end{tabular}

The cases discuss various behaviors of market agents in the market. One of the critical agents in the proposed approach is the wind producer which can affect the dispatch and the reserve requirement of the system. Therefore, different behavior of the wind agent in bidding in the market is studied. In table I, two types of bidding for the wind producer is shown. Each type represents a different behavior of the wind market agent in providing the bids. Note that the wind agent participates in the market with zero prices; hence, its bids in the market only include the quantity of wind production. The first type considers lack of accuracy in providing the wind scenarios (diverged bids), and the second type considers more converged manner in the offers. In both cases it is assumed that the expected value should be within a reasonable range. It should be noted that in Portugal, one agent is responsible for the aggregated participation of wind units in the market.

The results are obtained from the program using GAMS and CPLEX solver. The response time of the model is 23.758 seconds.

\section{1) Case I: Diverged Wind Offers:}

In this case, it is assumed that the market agent who is responsible for wind generating units submits diverged bids to the market. The bids from wind producers include different scenarios of power production assigned by a certain probability as shown in Table I. It is assumed that due to inaccuracy of agent's forecasts, the bids submitted to the market in this case show drastic changes in the amount of wind production in each scenario. As a result, they are called diverged bids. The results for the day-ahead dispatch of energy for hydro units and wind producer in both stochastic and deterministic approaches are shown in Fig. 1. As can be seen, major difference in the dispatch of these units occurs while using each of the market clearing approaches.

Based on Eq. (1) the activation of downward reserve counts as revenue in this problem; hence, in the stochastic approach, the market operator dispatches the wind unit on a lower amount which leads to mobilization of reserve and lower system costs. Consequently, when other scenarios of higher wind production occur, it will take benefit of the activation of downward reserve as it is available in the system. It indicates the difference of a hydro-penetrated system with the other ones. The reason is that the wind unit as a cheap resource in the system is competing with hydro units who also may participate in the market with zero prices. As a result, there is no difference for the market operator to choose between these two resources. On the other hand, the flexibility that the hydro units offer in the reserve market also has lower prices compared to flexible demand, demand response approaches, and other conventional units. 


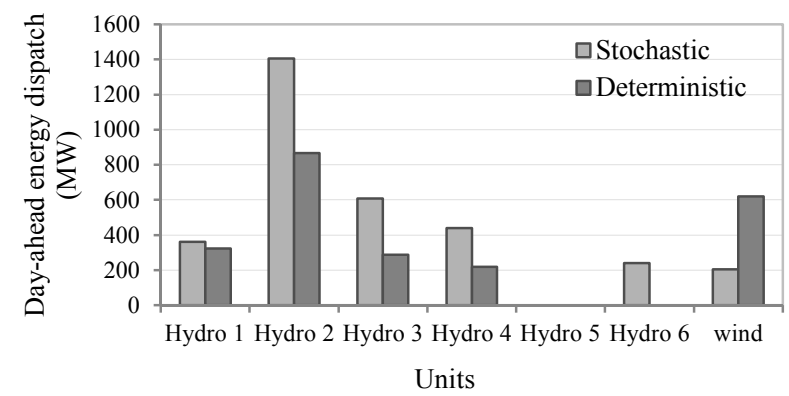

Figure 1. Day-ahead dispatch of hydro units and wind in stochastic and deterministic approach in Case I.

In Table II, the day-ahead revenue of each agent and their expected profit /penalty as well as the total system's cost are shown. From the results it can be deduced that with this type of bidding, the market results may not be fair for all the market participants. As for the wind agent, the implementation of the stochastic market clearing approach will lead to lower profit in the day-ahead and higher penalty due to the higher amount of deviation in different scenarios. It has been the initial intention of the proposed model to make the stochastic units economically viable for their uncertain behavior. However, it also suggests that the offers that the stochastic agent should make to the market can significantly affect its profit through the market.

On the other hand, for the hydro units, higher levels of profit are achieved in the day-ahead (due to higher clearing price) compared to the deterministic approach. Although some units such as Hydro II can encounter a penalty due to participating in the reserve mobilization, however, the total profit of the day-ahead and reserve is beneficial for the unit.

\section{2) Case II: Converged Wind Offers:}

In this case, type II of wind offers has been considered in the model and the results are shown in Fig. 2 and Table III. As can be seen in Fig. 2, the day-ahead dispatch of the two approaches (i.e., stochastic and deterministic) does not differ as it did in Case I. The reason is that the offers in this case are more converged to the expected value and do not change drastically. Therefore, other units are dispatched approximately the same as in the deterministic approach. In comparison to Case I, the levels of energy dispatched from the hydro units are also lower because of a higher share of wind unit in the day-ahead dispatch and lower level of reserve capacity requirement in case of other scenario's occurrence.

TABLE II. CASE I RESULTS

\begin{tabular}{|c|c|c|c|c|}
\hline & \multicolumn{2}{|c|}{ Stochastic } & \multicolumn{2}{|c|}{ Deterministic } \\
\hline & DA Rev. & $\begin{array}{c}\text { Exp. } \\
\text { Profit/Penalty }\end{array}$ & DA Rev. & $\begin{array}{c}\text { Exp. } \\
\text { Profit/Penalty }\end{array}$ \\
\hline Hydro I & $-20,694.01$ & - & $-15,584.40$ & - \\
\hline Hydro II & $-80,295.75$ & $3,048.8$ & $-41,669.03$ & -681.36 \\
\hline Hydro III & $-34,804.35$ & -721.44 & $-13,900.9$ & - \\
\hline Hydro IV & $-25,146$ & $-1,563.88$ & $-10,582$ & -102.01 \\
\hline Hydro V & 0 & - & 0 & - \\
\hline Hydro VI & $-13,716$ & -517.07 & 0 & - \\
\hline Wind & $-11,715.75$ & $-35,975.84$ & $-29,773.9$ & $2,392.5$ \\
\hline$\overline{\text { Total Cost. }}$ & \multicolumn{2}{|c|}{$\begin{array}{l}-921,220 \\
\end{array}$} & \multicolumn{2}{|c|}{ - $-614,400$} \\
\hline
\end{tabular}

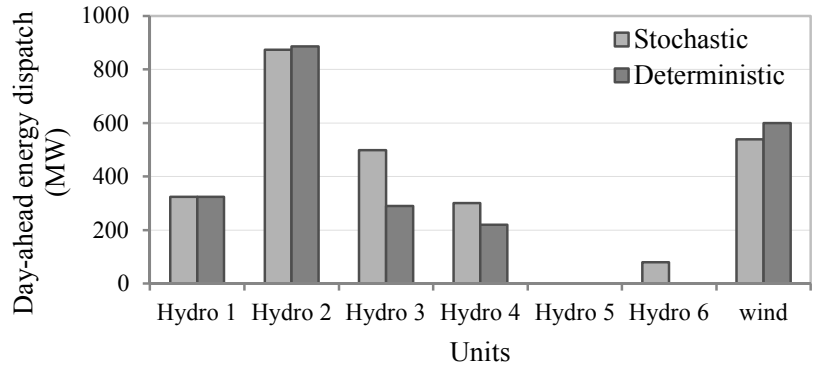

Figure 2. Day-ahead dispatch of hydro units and wind in stochastic and deterministic approach in Case II.

Referring to Table III, it can be seen that the profit made by the hydro units in this case due to lower deviation of the wind is also lower. The reason is that in this case, the wind unit is dispatched at $539 \mathrm{MW}$. Hence, if scenario \#1 occurs, there will be a lack of production and an increase of production is needed. In a hydro penetrated system such as in Portugal, the competitive power of thermal units are lower and there is always the chance of not being dispatched in the dayahead due to being more expensive. On the contrary, when these units participate in the reserve market, they will provide lower prices compared to hydro units. As a result, in the upward reserve mobilization, instead of using the hydro units, the market operator activates the reserve from thermal units. Moreover, as the amount of deviation is lower in this type of offer, the required amount of downward reserve is lower. Therefore, in this case, only hydro unit IV is activated in the reserve. From another point of view, it can be observed that the total system profit in this case is lower than Case I. It shows that in a flexible system inaccuracy in probabilistic bids in the market may lead to unfair distribution of profit between market participants.

TABLE III. CASE II RESUlTS

\begin{tabular}{|c|c|c||c|c|}
\cline { 2 - 5 } \multicolumn{1}{c|}{} & \multicolumn{2}{c||}{ Stochastic } & \multicolumn{2}{c|}{ Deterministic } \\
\cline { 2 - 5 } \multicolumn{1}{c|}{} & DA Rev. & $\begin{array}{c}\text { Exp. } \\
\text { Profit/Penalty }\end{array}$ & DA Rev. & $\begin{array}{c}\text { Exp. } \\
\text { Profit/Penalty }\end{array}$ \\
\hline Hydro I & $-15,840.36$ & - & $-15,584.4$ & - \\
\hline Hydro II & $-42,680.97$ & - & $-42,583$ & - \\
\hline Hydro III & $-24,396.11$ & - & $-13,900.9$ & - \\
\hline Hydro IV & $-14,667$ & -601.3 & $-10,582$ & -597.8 \\
\hline Hydro V & 0 & - & 0 & - \\
\hline Hydro VI & $-3,911.2$ & - & 0 & - \\
\hline \hline Wind & $-26,351.71$ & $2,342.3$ & $-28,860$ & $-1,236$ \\
\hline \hline Total Cost. & \multicolumn{3}{c|}{$-598,100$} & $-613,500$ \\
\hline
\end{tabular}

\section{CONCLUSION}

This paper has discussed the adaption of stochastic market modeling in a hydro-penetrated system. The proposed model includes the co-optimization of energy and reserve with considering the uncertainty of resources such as wind producers. The model was tested with the real data from Portuguese electricity market. In the data analysis, different schemes of wind units offers in the market have been studied.

The results showed that when dealing with a hydropenetrated network, the usual deductions from the proposed 
model cannot be totally relied on. The reason is that in a hydro-penetrated system, the necessary flexibility which is required for the market operator to compensate the uncertainty of wind is available with low prices. Unlike flexible demand or demand response programs, the hydro units are willing to decrease their production. Moreover, in comparison with flexible demand, the reduction of hydro units' production will have less cost imposed to the system. As a result, the market results and profits earned by the market participants will be highly affected by the probabilistic offers of wind agent. This may encourage the wind agents to use the diverged offers to make higher profit after the mobilization of reserve. As a result, a modification of the offers based on the wind forecast should be conducted by the market operator to avoid such cases.

\section{ACKNOWLEDGMENT}

This work is financed by the ERDF - European Regional Development Fund through the Operational Programme for Competitiveness and Internationalisation - COMPETE 2020 Programme, and by National Funds through the Portuguese funding agency, FCT - Fundação para a Ciência e a Tecnologia, within projects SAICTPAC/0004/2015- POCI-01-0145-FEDER-016434 and SusCity - MITPTB/CS/0026/2013.”

\section{REFERENCES}

[1] R. J. Kaye, H. R. Outhred, C. H. Bannister, "Forward contracts for the operation of an electricity industry under spot pricing," IEEE Trans. Power Systems, vol. 5, n.1, pp. 46-52, 1990.

[2] S. Wong, J. David Fuller, "Pricing energy and reserves using stochastic optimization in an alternative electricity market," IEEE Trans. Power Systems, vol. 22, n. 2, pp. 631-638, 2007.

[3] G. Pritchard, G. Zakeri, and A. Philpott, "A Single-Settlement, EnergyOnly Electric Power Market for Unpredictable and Intermittent Participants," Operations Research, vol. 58, n. 4-part-2, pp. 1210-1219, 2010.
[4] J. M. Morales, A. J. Conejo, K. Liu, and J. Zhong, "Pricing Electricity in Pools With Wind Producers," in IEEE Trans. Power Systems, vol. 27, no. 3, pp. 1366-1376, Aug. 2012.

[5] J. Khazaei, G. Zakeri, SS Oren. "Market clearing mechanisms under demand uncertainty," Tech. rep., Electric Power Optimization Centre, University of Auckland, 2013.

[6] J. M. Morales, M. Zugno, S. Pineda, and P. Pinson, "Electricity market clearing with improved scheduling of stochastic production," European Journal of Operational Research, Vol. 235, Issue 3, pp. 765-774, June 2014.

[7] L. Baringo and A. J. Conejo, "Offering Strategy of Wind-Power Producer: A Multi-Stage Risk-Constrained Approach," IEEE Trans. Power Systems, vol. 31, no. 2, pp. 1420-1429, March 2016.

[8] Y. Zhang and G. B. Giannakis, "Distributed Stochastic Market Clearing With High-Penetration Wind Power," in IEEE Trans. Power Systems, vol. 31, no. 2, pp. 895-906, March 2016.

[9] N. Zhang et al., "A Convex Model of Risk-Based Unit Commitment for Day-Ahead Market Clearing Considering Wind Power Uncertainty," in IEEE Trans. Power Systems, vol. 30, no. 3, pp. 1582-1592, May 2015.

[10] W. A. Bukhsh, A. Papakonstantinou and P. Pinson, "A robust optimisation approach using CVaR for unit commitment in a market with probabilistic offers," 2016 IEEE International Energy Conference (ENERGYCON), Leuven, 2016, pp. 1-6.

[11] F. Abbaspourtorbati, A. Conejo, J. Wang, and R. Cherkaoui, "Pricing Electricity through a Stochastic Non-Convex Market-Clearing Model," IEEE Trans. Power Systems, doi: 10.1109/TPWRS.2016.2569533.

[12] S. Martín, Y. Smeers and J. A. Aguado, "A Stochastic Two Settlement Equilibrium Model for Electricity Markets With Wind Generation," IEEE Trans. Power Systems, vol. 30, no. 1, pp. 233-245, Jan. 2015.

[13] F. Abbaspourtorbati and M. Zima, "The Swiss Reserve Market: Stochastic Programming in Practice," IEEE Trans. Power Systems, vol. 31, no. 2, pp. 1188-1194, March 2016.

[14] F. Abbaspourtorbati, A. J. Conejo, J. Wang, and R. Cherkaoui, "Is Being Flexible Advantageous for Demands?" IEEE Trans. Power Systems, DOI: 10.1109/TPWRS.2016.2606606.

[15] REN Energy Market Information system, Market information and results, available online at: http://www.mercado.ren.pt/.

[16] MIBEL Market Data and information, available online at: http://www.omie.es/. 